\title{
ON NILPOTENT FILIFORM LIE ALGEBRAS OF DIMENSION EIGHT
}

\section{P. BARBARI and A. KOBOTIS}

Received 1 December 2001

\begin{abstract}
The aim of this paper is to determine both the Zariski constructible set of characteristically nilpotent filiform Lie algebras $g$ of dimension 8 and that of the set of nilpotent filiform Lie algebras whose group of automorphisms consists of unipotent automorphisms, in the variety of filiform Lie algebras of dimension 8 over $C$.
\end{abstract}

2000 Mathematics Subject Classification: 17B30.

1. Introduction. Characteristically nilpotent Lie algebras were defined by Dixmier and Lister in [4] and filiform Lie algebras by Vergne in [8]. A complete classification of nilpotent filiform Lie algebras of dimension 8 is available since 1988 in [1] due to Ancochéa-Bermúdez and Goze. Then, Echarte-Reula et al. [6], considering that a filiform Lie algebra $g$ is characteristically nilpotent if and only if $g$ is not a derived algebra, obtained a list of characteristically nilpotent filiform Lie algebras of dimension 8. Recently, Castro-Jiménez and Núñez-Valdés studied extensively in [2,3] the cases of dimension 9 and 10 and gave the sets of the corresponding characteristically nilpotent Lie algebras as a finite union of Zariski locally closed subsets. In 1970, Dyer in [5] gave an example of a characteristically nilpotent Lie algebra of dimension 9 and showed that each automorphism of this Lie algebra is unipotent. Some years later, Favre in [7] reached the same result working on an example of a characteristically nilpotent Lie algebra of dimension 7.

In this paper, we study the Lie algebras of dimension 8 . We first express the set of characteristically nilpotent filiform Lie algebras $g$ as a finite union of locally closed subsets, then we prove that the set of nilpotent filiform Lie algebras $g$, whose group of automorphisms consists of unipotent automorphisms, is a Zariski constructible set in the variety of nilpotent filiform Lie algebras, and we express it as a finite union of locally closed subsets. Furthermore, we prove that the group of automorphisms $\operatorname{Aut}(g)$ of each one of the above characteristically nilpotent filiform Lie algebras consists of unipotent automorphisms except two, in each of which the set of their unipotent automorphisms forms a proper subgroup of the group $\operatorname{Aut}(g)$.

2. Preliminaries. Let $g$ be a Lie algebra of dimension $n$ over $C$ of characteristic zero. If we consider the descending central sequence $C^{1} g=g$, 
$C^{2} g=[g, g], \ldots$, and $C^{q} g=\left[g, C^{q-1} g\right], \ldots$, of the above Lie algebra, then the Lie algebra $g$ is called filiform if $\operatorname{dim}_{C} C^{q} g=n-q$ for $2 \leq q \leq n$ [8].

Let $g$ be a filiform Lie algebra of dimension $n$. Then, there exists a basis $E=\left\{e_{1}, e_{2}, \ldots, e_{n}\right\}$ of $g$ such that $e_{1} \in g \backslash C^{2} g$, the matrix of $\operatorname{ad}\left(e_{1}\right)$ with respect to $E$ has a Jordan block of order $n-1$, and $C^{i} g$ is the vector space generated by $\left\{e_{2}, e_{3}, \ldots, e_{n-i+1}\right\}$ with $2 \leq i \leq n-1$. Such a basis is called an adapted basis.

Let $W$ be a vector space over a field $C$ of dimension $n$. A subset $A$ of $W$ is an algebraic one if there exists a set $B$ of polynomial functions on $W$ such that $A=$ $\{x \in W / p(x)=0$, for all $p \in B\}$. We consider the set $C[x]$ of all polynomials in $n$ variables $x=\left\{x_{1}, \ldots, x_{n}\right\}$ over $C$ and $I$ an ideal of $C[x]$. We denote by $\mathscr{V}(I)$ the set $\mathscr{V}(I)=\left\{a \in C^{n} / p(a)=0\right.$, for all $\left.p \in I\right\}$. As a consequence of the above definitions, $\mathscr{V}(I)$ is an algebraic subset of the vector space $C^{n}$ and so the Zariski topology on the space $C^{n}$ is the one whose closed sets are $\mathscr{V}(I)$. Finally, we denote by $D(I)$ the complement of $\mathscr{V}(I)$ in $C^{n}$.

3. The equations. It has been proved in [1] that there exists a basis $\left\{e_{1}, e_{2}\right.$, $\left.\ldots, e_{8}\right\}$ such that every nilpotent filiform Lie algebra $g$ over a field $C$ of characteristic zero of dimension 8 is isomorphic to one of the Lie algebras belonging to the nine-parameter family given in [1].

We now consider a change of the previous base of the nilpotent filiform Lie algebra $g$ such that $Y_{i}=e_{i}, i=1,2, \ldots, 7$, and $Y_{8}=e_{8}+a_{1} e_{1}$. So, the set of nilpotent filiform Lie algebras over $C$ can be parametrized by the points $\left(a_{2}, a_{3}, \ldots, a_{9}\right)$ of the algebraic set $V^{\prime} \in C^{8}$, and the above-mentioned equations of the nine-parameter family, with respect to the new base $A=\left\{e_{1}, e_{2}, \ldots\right.$, $\left.e_{8}\right\}$, takes the form

$$
\begin{aligned}
& {\left[e_{1}, e_{i}\right]=e_{i-1}, \quad i \geq 3,} \\
& {\left[e_{4}, e_{7}\right]=a_{2} e_{2},} \\
& {\left[e_{4}, e_{8}\right]=a_{2} e_{3}+a_{3} e_{2},} \\
& {\left[e_{5}, e_{6}\right]=a_{4} e_{2},} \\
& {\left[e_{5}, e_{7}\right]=\left(a_{2}+a_{4}\right) e_{3}+a_{5} e_{2},} \\
& {\left[e_{5}, e_{8}\right]=\left(2 a_{2}+a_{4}\right) e_{4}+\left(a_{3}+a_{5}\right) e_{3}+a_{6} e_{2},} \\
& {\left[e_{6}, e_{7}\right]=\left(a_{2}+a_{4}\right) e_{4}+a_{5} e_{3}+a_{7} e_{2},} \\
& {\left[e_{6}, e_{8}\right]=\left(3 a_{2}+2 a_{4}\right) e_{5}+\left(a_{3}+2 a_{5}\right) e_{4}+\left(a_{6}+a_{7}\right) e_{3}+a_{8} e_{2},} \\
& {\left[e_{7}, e_{8}\right]=\left(3 a_{2}+2 a_{4}\right) e_{6}+\left(a_{3}+2 a_{5}\right) e_{5}+\left(a_{6}+a_{7}\right) e_{4}+a_{8} e_{3}+a_{9} e_{2},}
\end{aligned}
$$

with $a_{j} \in C, j=2,3, \ldots, 9$, verifying the equations

$$
\begin{gathered}
a_{2}+a_{4}=0, \\
a_{2}\left(5 a_{5}+2 a_{3}\right)=0 .
\end{gathered}
$$

Those two equations are consequences of the Jacobi's identities. 
4. Characteristically nilpotent filiform Lie algebras. Let $g$ be a nilpotent Lie algebra of dimension $n$ over $C$ of characteristic zero. A Lie algebra $g$ is said to be characteristically nilpotent if the Lie algebra of its derivations $\mathrm{D}$ is nilpotent. By $\mathrm{D}: g \rightarrow g$ verifying $\mathrm{D}[x, y]=[\mathrm{D} x, y]+[x, \mathrm{D} y]$ for all $(x, y) \in g$, we mean a derivation of $g$.

Let $\mathrm{D}=\left(d_{i j}\right) \in \operatorname{Mat}(8 \times 8, C)$ be the set of matrices representing the derivations $\mathrm{D}$ of the filiform Lie algebras over $C$ of dimension 8 with respect to the new base $A=\left\{e_{1}, e_{2}, \ldots, e_{8}\right\}$.

Suppose that

$$
\begin{gathered}
\mathrm{D} e_{k}=\sum d_{k \lambda} e_{\lambda}, \quad 1 \leq k, \lambda \leq 8, d_{k \lambda} \in C, \\
\mathrm{D}\left[e_{i}, e_{j}\right]-\mathrm{D} e_{k}=0, \quad 1 \leq i<j \leq 8,1 \leq k \leq 8 .
\end{gathered}
$$

From

$$
\mathrm{D}\left[e_{1}, e_{2}\right]=0, \quad \mathrm{D}\left[e_{1}, e_{i}\right]=\mathrm{D} e_{i-1}, \quad i \geq 3,
$$

we deduce that

$$
d_{i j}=0, \quad 2 \leq i \leq 7, i<j, 3 \leq j \leq 8, \quad d_{i 1}=0, \quad 2 \leq i \leq 8 .
$$

For each $(i, j, k), 1 \leq i<j \leq 8,1 \leq k \leq 8$, we denote by $b(i, j, k)$ the coefficient of $e_{k}$ in the expression $\mathrm{D}\left[e_{i}, e_{j}\right]-\left[\mathrm{D} e_{i}, e_{j}\right]-\left[e_{i}, \mathrm{D} e_{j}\right]$ with respect to the base $A$. From above, we obtain a homogeneous linear system defined by

$$
S=\{b(i, j, k)=0,1 \leq i<j \leq 8,1 \leq k \leq 8\} .
$$

The solutions satisfying system (4.4) are elements of the set of matrices $\mathrm{D}=\left(d_{i j}\right) \in \operatorname{Mat}(8 \times 8, C)$.

In case that $\mathrm{D}$ are nilpotent matrices, according to the previous definition, the filiform Lie algebra $g$ is characteristically nilpotent.

4.1. The system of equations. Let $t=\left(a_{2}, a_{3}, a_{5}, a_{6}, a_{7}, a_{8}, a_{9}\right)$ be a point of $V \in C^{7}, g_{t}$ the corresponding filiform Lie algebra of dimension 8 , and $S_{t}$ the homogeneous linear system corresponding to (4.4). We consider the linear system $S_{t}$ as a system with coefficients in the quotient ring $R / I$ where $R=$ $C\left[a_{2}, a_{3}, a_{5}, a_{6}, a_{7}, a_{8}, a_{9}\right]$ and $I$ is the ideal generated by (3.2). In that case, 
system $S$ in (4.4) is reduced to the following equivalent system $S_{t}$ :

$$
\begin{aligned}
& d_{11}-d_{22}+d_{33}=0 \\
& d_{11}-d_{77}+d_{88}=0 \\
& a_{2} d_{17}-d_{76}+d_{87}=0 \\
& a_{2} d_{22}-a_{2} d_{44}-a_{2} d_{77}=0 \\
& a_{2} d_{22}-a_{2} d_{55}-a_{2} d_{66}=0 \\
& a_{2} d_{33}-a_{2} d_{44}-a_{2} d_{88}=0 \\
& a_{5} d_{33}-a_{5} d_{66}-a_{5} d_{77}=0 \\
& a_{2} d_{44}-a_{2} d_{55}-a_{2} d_{88}=0 \\
& a_{2} d_{55}-a_{2} d_{66}-a_{2} d_{88}=0 \\
& a_{2} d_{66}-a_{2} d_{77}-a_{2} d_{88}=0 \\
& d_{11}-a_{2} d_{18}-d_{33}+d_{44}=0 \\
& d_{11}-a_{2} d_{18}-d_{44}+d_{55}=0 \\
& d_{11}-a_{2} d_{18}-d_{55}+d_{66}=0 \\
& d_{11}-a_{2} d_{18}-d_{66}+d_{77}=0 \\
& \left(a_{3}+a_{5}\right) d_{18}+d_{43}-d_{54}=0 \\
& \left(a_{6}+a_{7}\right) d_{18}+d_{64}-d_{75}=0 \\
& \left(a_{3}+2 a_{5}\right) d_{18}+d_{54}-d_{65}=0 \\
& \left(a_{3}+2 a_{5}\right) d_{18}+d_{65}-d_{76}=0 \\
& a_{5} d_{16}-a_{8} d_{18}-d_{63}+d_{74}=0 \\
& a_{2} d_{17}+a_{3} d_{18}+d_{32}-d_{43}=0 \\
& a_{5} d_{17}+\left(a_{6}+a_{7}\right) d_{18}+d_{53}-d_{64}=0 \\
& a_{2} d_{15}+a_{7} d_{17}+a_{8} d_{18}+d_{52}-d_{63}=0 \\
& a_{2} d_{16}-a_{5} d_{17}-a_{6} d_{18}-d_{42}+d_{53}=0 \\
& a_{2} d_{16}+\left(a_{3}+2 a_{5}\right) d_{17}-d_{75}+d_{86}=0 \\
& a_{3} d_{22}+a_{2} d_{32}-a_{3} d_{44}-a_{2} d_{87}-a_{3} d_{88}=0 \\
& a_{5} d_{22}-a_{2} d_{54}+a_{5} d_{55}+a_{2} d_{76}+a_{5} d_{77}=0 \\
& a_{2} d_{14}+a_{5} d_{15}+a_{7} d_{16}-a_{9} d_{18}-d_{62}+d_{73}=0 \\
& a_{3} d_{14}+a_{6} d_{15}+a_{8} d_{16}+a_{9} d_{17}-d_{72}+d_{83}=0 \\
& \left(a_{3}+a_{5}\right) d_{15}+\left(a_{6}+a_{7}\right) d_{16}+a_{8} d_{17}-d_{73}+d_{84}=0 \\
& a_{2} d_{15}+\left(a_{3}+2 a_{5}\right) d_{16}+\left(a_{6}+a_{7}\right) d_{17}-d_{74}+d_{85}=0
\end{aligned}
$$




$$
\begin{aligned}
& a_{7} d_{22}+a_{5} d_{32}-a_{2} d_{64}-a_{5} d_{65}-a_{7} d_{66}-a_{2} d_{75}-a_{7} d_{77}=0 \\
& \left(a_{3}+a_{5}\right) d_{33}+a_{2} d_{43}-a_{2} d_{54}-\left(a_{3}+a_{5}\right) d_{55}-\left(a_{3}+a_{5}\right) d_{88}=0 \\
& \left(a_{3}+2 a_{5}\right) d_{44}+a_{2} d_{54}-a_{2} d_{65}-\left(a_{3}+2 a_{5}\right) d_{66}-\left(a_{3}+2 a_{5}\right) d_{88}=0 \\
& \left(a_{3}+2 a_{5}\right) d_{55}+a_{2} d_{65}-a_{2} d_{76}-\left(a_{3}+2 a_{5}\right) d_{77}-\left(a_{3}+2 a_{5}\right) d_{88}=0 \\
& a_{6} d_{22}+\left(a_{3}+a_{5}\right) d_{32}+a_{2} d_{42}-a_{3} d_{54}-a_{6} d_{55}+a_{2} d_{86}-a_{5} d_{87}-a_{6} d_{88}=0 \\
& \left(a_{6}+a_{7}\right) d_{33}+\left(a_{3}+2 a_{5}\right) d_{43}+a_{2} d_{53}-a_{2} d_{64}-\left(a_{3}+a_{5}\right) d_{65} \\
& \quad-\left(a_{6}+a_{7}\right) d_{66}-a_{5} d_{87}-\left(a_{6}+a_{7}\right) d_{88}=0 \\
& \quad\left(a_{6}+a_{7}\right) d_{44}+\left(a_{3}+2 a_{5}\right) d_{54}+a_{2} d_{64}-a_{2} d_{75}-\left(a_{3}+2 a_{5}\right) d_{76} \\
& \quad-\left(a_{6}+a_{7}\right) d_{77}-\left(a_{6}+a_{7}\right) d_{88}=0 \\
& a_{8} d_{22}+\left(a_{6}+a_{7}\right) d_{32}+\left(a_{3}+2 a_{5}\right) d_{42}+a_{2} d_{52}-a_{3} d_{64}-a_{6} d_{65} \\
& \quad-a_{8} d_{66}-a_{2} d_{85}-a_{7} d_{87}-a_{8} d_{88}=0 \\
& a_{8} d_{33}+\left(a_{6}+a_{7}\right) d_{43}+\left(a_{3}+2 a_{5}\right) d_{53}+a_{2} d_{63}-a_{2} d_{74}-\left(a_{3}+a_{5}\right) d_{75} \\
& \quad-\left(a_{6}+a_{7}\right) d_{76}-a_{8} d_{77}+a_{5} d_{86}-a_{8} d_{88}=0 \\
& a_{9} d_{22}+a_{8} d_{32}+\left(a_{6}+a_{7}\right) d_{42}+\left(a_{3}+2 a_{5}\right) d_{52}+a_{2} d_{62}-a_{3} d_{74}-a_{6} d_{75} \\
& \quad-a_{8} d_{76}-a_{9} d_{77}+a_{2} d_{84}+a_{5} d_{85}+a_{7} d_{86}-a_{9} d_{88}=0 .
\end{aligned}
$$

The solutions satisfying $S_{t}$ are derivations of the nilpotent filiform Lie algebra $g_{t}$. If all the derivations of $g_{t}$ are nilpotent, then $g_{t}$ is characteristically nilpotent.

We will prove that the set of points $t \in V \subset C^{7}$, such that there exists a solution of $S_{t}$ satisfying the conditions of $g_{t}$ being a characteristically nilpotent filiform Lie algebra, is a Zariski constructible set, and we will express it as a finite union of Zariski locally closed subsets. To realize the above idea, we study $S_{t}$ in suitable subsets of $V$.

4.2. Main results. We consider two cases: first, $a_{2} \neq 0$ and then, $a_{2}=0$.

4.2.1. $a_{2} \neq 0$. Let the open set $V \cap D\left(a_{2}\right)$. Because of the equation $a_{2}\left(5 a_{5}+\right.$ $\left.2 a_{3}\right)=0$, we can distinguish the following two subcases.

(1) $\left(a_{3} \neq 0\right)$. First, we consider the set $T^{(1)}=V \cap D\left(a_{2} \cdot a_{3}\right)$. From $5 a_{5}+2 a_{3}=$ 0 , we obtain $a_{5}=-(2 / 5) a_{3}$. By doing the necessary calculations in system $S_{t}$, we prove that, in the set of points $T^{(1)} \cap D\left(Q_{1}\right)$ with $Q_{1}=2 a_{3}^{2}-25 a_{2} a_{6}-$ $25 a_{2} a_{7}$, the corresponding Lie algebra is characteristically nilpotent.

(2) $\left(a_{3}=0\right)$. Now, we consider the set $T^{(2)}=V \cap D\left(a_{2}\right) \cap \mathscr{V}\left(a_{3}\right)$. From $5 a_{5}+$ $2 a_{3}=0$, we obtain $a_{5}=0$. In case that $a_{6}+a_{7} \neq 0$ and $a_{8} \neq 0$, that means in $T^{(2)} \cap D\left(\left(a_{6}+a_{7}\right) \cdot a_{8}\right)$, only one Lie algebra is characteristically nilpotent.

From the above, we can state the following theorem. 
THEOREM 4.1. Consider the set of complex filiform Lie algebras. Consider $C^{8}$ with $\left(a_{2}, a_{3}, \ldots, a_{9}\right)$ as coordinates given by (3.1) and let $V$ be the hypersurface defined in $C^{7}$ by (3.2). In the Zariski open set $V \cap D\left(a_{2}\right)$, the Zariski constructible subset of characteristically nilpotent Lie algebras is defined as the union of the following subsets:

$$
\begin{gathered}
D\left(a_{3} \cdot\left(2 a_{3}^{2}-25 a_{2} a_{6}-25 a_{2} a_{7}\right)\right), \\
\mathscr{V}\left(a_{3}\right) \cap D\left(\left(a_{6}+a_{7}\right) \cdot a_{8}\right) .
\end{gathered}
$$

4.2.2. $a_{2}=0$. We consider the set $T^{(3)}=V \cap \mathscr{V}\left(a_{2}\right)$. Because of the equation $a_{2}\left(5 a_{5}+2 a_{3}\right)=0$, we can distinguish the following subcases.

(1) $\left(a_{5} \neq 0\right)$. So, we obtain the set $T^{(3)} \cap D\left(a_{5}\right)$ and we distinguish the following:

(1A) $\left(a_{3}+2 a_{5} \neq 0\right)$. In the subset $T^{(3)} \cap D\left(a_{5} \cdot\left(a_{3}+2 a_{5}\right) \cdot Q_{2}\right)$ with $Q_{2}=$ $2 a_{3}^{2} a_{7}-3 a_{3} a_{5} a_{6}+5 a_{3} a_{5} a_{7}-3 a_{5}^{2} a_{6}+5 a_{5}^{2} a_{7}$, the corresponding Lie algebra is characteristically nilpotent,

(1B) $\left(a_{3}+2 a_{5}=0\right)$. The corresponding Lie algebra in the set of points $T^{(3)} \cap$ $D\left(a_{5} \cdot\left(a_{6}+a_{7}\right)\right) \cap \mathscr{V}\left(a_{3}+2 a_{5}\right)$ is characteristically nilpotent.

(2) $\left(a_{5}=0\right)$. First, we distinguish two subcases $a_{3} \neq 0$ and $a_{3}=0$.

(2A) $\left(a_{3} \neq 0\right)$. Then, we consider the set $T^{(3)} \cap \mathscr{V}\left(a_{5}\right) \cap D\left(a_{3}\right)$. By doing some calculations, we distinguish two more subcases.

(i) $\left(a_{7} \neq 0\right)$. In this case, the Lie algebra corresponding to the set of points $T^{(3)} \cap \mathscr{V}\left(a_{5}\right) \cap D\left(a_{3} \cdot a_{7}\right)$ is characteristically nilpotent.

(ii) $\left(a_{7}=0\right)$. Now, we study $S_{t}$ in the set of points $Z=T^{(3)} \cap \mathscr{V}\left(a_{5}, a_{7}\right) \cap D\left(a_{3}\right)$. The Lie algebras corresponding to the set of points $Z \cap\left(D\left(Q_{31}\right) \cup D\left(Q_{32}\right)\right)$, with $Q_{31}=4 a_{3} a_{8}-5 a_{6}^{2}$ and $Q_{32}=2 a_{3}^{2} a_{9}-2 a_{3} a_{6} a_{8}-a_{6}^{3}$, are characteristically nilpotent.

(2B) $\left(a_{3}=0\right)$. We operate in $T^{(3)} \cap \mathscr{V}\left(a_{3}, a_{5}\right)$ and we distinguish the cases $a_{7} \neq 0$ and $a_{7}=0$.

(i) $\left(a_{7} \neq 0\right)$. The Lie algebra corresponding to the set of points $T^{(3)} \cap \mathscr{V}\left(a_{3}, a_{5}\right)$ $\cap D\left(a_{7} \cdot a_{8}\right)$ is characteristically nilpotent.

(ii) $\left(a_{7}=0\right)$. We now consider the subset $T^{(3)} \cap \mathscr{V}\left(a_{3}, a_{5}, a_{7}\right)$. We distinguish another two subcases, $a_{6} \neq 0$ and $a_{6}=0$.

(iiA) $\left(a_{6} \neq 0\right)$. The Lie algebra corresponding to $T^{(3)} \cap \mathscr{V}\left(a_{3}, a_{5}, a_{7}\right) \cap D\left(a_{6}\right.$. $\left.a_{8}\right)$ is characteristically nilpotent.

(iiB) $\left(a_{6}=0\right)$. The Lie algebra in the set $T^{(3)} \cap \mathscr{V}\left(a_{3}, a_{5}, a_{6}, a_{7}\right) \cap D\left(a_{8} \cdot a_{9}\right)$ is characteristically nilpotent.

So, we have proved the following theorem.

THEOREM 4.2. Consider the set of complex filiform Lie algebras. Consider $C^{8}$ with $\left(a_{2}, a_{3}, \ldots, a_{9}\right)$ as coordinates given by (3.1), and let $V$ be the hypersurface defined in $C^{7}$ by (3.2). The Zariski constructible subset of characteristically nilpotent Lie algebras in the Zariski closed set $V \cap \mathscr{V}\left(a_{2}\right)$ is defined as the union 
of the following subsets:

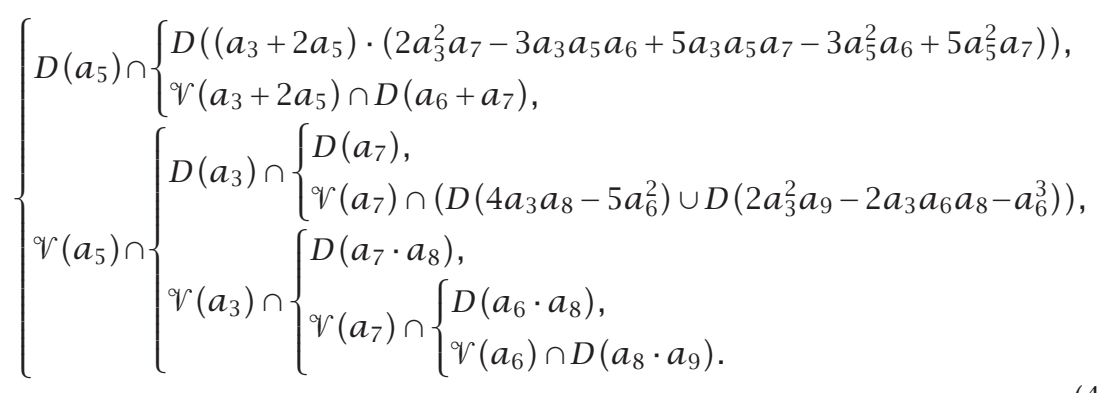

By $(\{)$, we mean the union of the corresponding sets.

5. Unipotent automorphisms of nilpotent filiform Lie algebras. Let $g$ be a nilpotent Lie algebra of dimension $n$ over $C$ of characteristic zero. The automorphism $\theta$ of a Lie algebra $g$ over $C$ is defined by the mapping $[x, y] \rightarrow$ $\theta([x, y])=[\theta(x), \theta(y)]$ for all $(x, y) \in g$. An automorphism $\theta$ is called unipotent if its representation, with respect to the base $\left\{e_{1}, e_{2}, \ldots, e_{n}\right\}$, has the form

$$
B=\left(b_{i j}\right) \in \operatorname{Mat}(n \times n, C), \quad b_{i j}=0, j<i, b_{i i}=1,1 \leq i, j \leq n .
$$

Let $B=\left(b_{i j}\right) \in \operatorname{Mat}(8 \times 8, C)$ be the set of matrices representing the automorphisms $\theta$ of the filiform Lie algebras over $C$ of dimension 8 with respect to the new base $A=\left\{e_{1}, e_{2}, \ldots, e_{8}\right\}$.

Suppose that

$$
\begin{gathered}
\theta\left(e_{k}\right)=\sum b_{k \lambda} e_{\lambda}, \quad 1 \leq k, \lambda \leq 8, b_{k \lambda} \in C, \\
\theta\left(\left[e_{i}, e_{j}\right]\right)-\theta\left(e_{k}\right)=0, \quad 1 \leq i<j \leq 8,1 \leq k \leq 8 .
\end{gathered}
$$

From

$$
\theta\left(\left[e_{1}, e_{2}\right]\right)=0, \quad \theta\left(\left[e_{1}, e_{i}\right]\right)=\theta\left(e_{i-1}\right), \quad i \geq 3, \quad \theta\left(\left[e_{3}, e_{8}\right]\right)=0,
$$

we deduce that

$$
b_{i j}=0, \quad 3 \leq i \leq 8, j<i, 2 \leq j \leq 7, \quad b_{1 j}=0, \quad 2 \leq j \leq 8 .
$$

For each $(i, j, k), 1 \leq i<j \leq 8,1 \leq k \leq 8$, we denote by $c(i, j, k)$ the coefficient of $e_{k}$ in the expression $\theta\left(\left[e_{i}, e_{j}\right]\right)-\left[\theta\left(e_{i}\right), \theta\left(e_{j}\right)\right]$ with respect to the base $A$.

From the above, we obtain a homogeneous system defined by

$$
S^{\prime}=\{c(i, j, k)=0,1 \leq i<j \leq 8,1 \leq k \leq 8\}
$$


The solutions satisfying system (5.5) are elements of the set of matrices $B=\left(b_{i j}\right) \in \operatorname{Mat}(8 \times 8, C)$.

In case that $B=\left(b_{i j}\right) \in \operatorname{Mat}(8 \times 8, C)$ are matrices of the form (5.1), according to the above definition, the group of automorphisms $\operatorname{Aut}(g)$ of the corresponding filiform Lie algebra $g$ consists of unipotent automorphisms.

5.1. The system of equations. Let $t=\left(a_{2}, a_{3}, a_{5}, a_{6}, a_{7}, a_{8}, a_{9}\right)$ be a point of $V \in C^{7}, g_{t}$ the corresponding filiform Lie algebra of dimension 8 , and $S_{t}^{\prime}$ the homogeneous system corresponding to (5.5). We will consider the linear system $S_{t}^{\prime}$ as a system with coefficients in the quotient ring $R / I$ where $R=$ $C\left[a_{2}, a_{3}, a_{5}, a_{6}, a_{7}, a_{8}, a_{9}\right]$ and $I$ is the ideal generated by (3.2). In that case, system $S^{\prime}$ in (5.5) is reduced to the following equivalent system $S_{t}^{\prime}$ :

$$
\begin{aligned}
& b_{22}-b_{11} b_{33}=0 \\
& b_{77}-b_{11} b_{88}=0 \\
& a_{2} b_{22}-a_{2} b_{44} b_{77}=0 \\
& a_{2} b_{22}-a_{2} b_{55} b_{66}=0 \\
& a_{2} b_{33}-a_{2} b_{44} b_{88}=0 \\
& a_{2} b_{44}-a_{2} b_{55} b_{88}=0 \\
& a_{2} b_{55}-a_{2} b_{66} b_{88}=0 \\
& a_{2} b_{66}-a_{2} b_{77} b_{88}=0 \\
& a_{5} b_{33}-a_{5} b_{66} b_{77}=0 \\
& b_{33}-b_{11} b_{44}+a_{2} b_{44} b_{81}=0 \\
& b_{44}-b_{11} b_{55}+a_{2} b_{55} b_{81}=0 \\
& b_{55}-b_{11} b_{66}+a_{2} b_{66} b_{81}=0 \\
& b_{66}-b_{11} b_{77}+a_{2} b_{77} b_{81}=0 \\
& b_{23}-b_{11} b_{34}+a_{2} b_{44} b_{71}+a_{3} b_{44} b_{81}=0 \\
& b_{67}-b_{11} b_{78}-a_{2} b_{71} b_{88}+a_{2} b_{78} b_{81}=0 \\
& a_{3} b_{22}+a_{2} b_{23}-a_{2} b_{44} b_{78}-a_{3} b_{44} b_{88}=0 \\
& a_{5} b_{22}-a_{2} b_{45} b_{77}+a_{2} b_{55} b_{67}-a_{5} b_{55} b_{77}=0 \\
& b_{34}-b_{11} b_{45}+a_{2} b_{45} b_{81}+\left(a_{3}+a_{5}\right) b_{55} b_{81}=0 \\
& b_{45}-b_{11} b_{56}+a_{2} b_{56} b_{81}+\left(a_{3}+2 a_{5}\right) b_{66} b_{81}=0 \\
& b_{56}-b_{11} b_{67}+a_{2} b_{67} b_{81}+\left(a_{3}+2 a_{5}\right) b_{77} b_{81}=0 \\
& \left(a_{3}+a_{5}\right) b_{33}+a_{2} b_{34}-a_{2} b_{45} b_{88}-\left(a_{3}+a_{5}\right) b_{55} b_{88}=0 \\
& \left(a_{3}+2 a_{5}\right) b_{44}+a_{2} b_{45}-a_{2} b_{56} b_{88}-\left(a_{3}+2 a_{5}\right) b_{66} b_{88}=0 \\
& \left(a_{3}+2 a_{5}\right) b_{55}+a_{2} b_{56}-a_{2} b_{67} b_{88}-\left(a_{3}+2 a_{5}\right) b_{77} b_{88}=0 \\
& b_{46}-b_{11} b_{57}+a_{2} b_{57} b_{81}+\left(a_{3}+2 a_{5}\right) b_{67} b_{81}+\left(a_{6}+a_{7}\right) b_{77} b_{81}=0 \\
& b_{24}-b_{11} b_{35}+a_{2} b_{45} b_{71}+a_{3} b_{45} b_{81}-a_{2} b_{55} b_{61}+a_{5} b_{55} b_{71}+a_{6} b_{55} b_{81}=0 \\
& { }_{1}
\end{aligned}
$$




$$
\begin{aligned}
& a_{7} b_{22}+a_{5} b_{23}-a_{2} b_{46} b_{77}+a_{2} b_{56} b_{67}-a_{5} b_{56} b_{77}-a_{2} b_{57} b_{66}-a_{7} b_{66} b_{77}=0 \\
& b_{35}-b_{11} b_{46}+a_{2} b_{46} b_{81}+\left(a_{3}+a_{5}\right) b_{56} b_{81}+a_{5} b_{66} b_{71}+\left(a_{6}+a_{7}\right) b_{66} b_{81}=0 \\
& b_{57}-b_{11} b_{68}-a_{2} b_{61} b_{88}+a_{2} b_{68} b_{81}-\left(a_{3}+2 a_{5}\right) b_{71} b_{88}+\left(a_{3}+2 a_{5}\right) b_{78} b_{81}=0 \\
& \left(a_{6}+a_{7}\right) b_{44}+\left(a_{3}+2 a_{5}\right) b_{45}+a_{2} b_{46}-a_{2} b_{57} b_{88}-\left(a_{3}+2 a_{5}\right) b_{67} b_{88} \\
& -\left(a_{6}+a_{7}\right) b_{77} b_{88}=0 \\
& b_{36}-b_{11} b_{47}+a_{2} b_{47} b_{81}+\left(a_{3}+a_{5}\right) b_{57} b_{81}-a_{5} b_{61} b_{77}+a_{5} b_{67} b_{71} \\
& +\left(a_{6}+a_{7}\right) b_{67} b_{81}+a_{8} b_{77} b_{81}=0 \\
& a_{6} b_{22}+\left(a_{3}+2 a_{5}\right) b_{23}+a_{2} b_{24}-a_{2} b_{45} b_{78}-a_{3} b_{45} b_{88}+a_{2} b_{55} b_{68} \\
& -a_{5} b_{55} b_{78}-a_{6} b_{55} b_{88}=0 \\
& \left(a_{6}+a_{7}\right) b_{33}+\left(a_{3}+2 a_{5}\right) b_{34}+a_{2} b_{35}-a_{2} b_{46} b_{88}-\left(a_{3}+a_{5}\right) b_{56} b_{88} \\
& -a_{5} b_{66} b_{78}-\left(a_{6}+a_{7}\right) b_{66} b_{88}=0 \\
& b_{25}-b_{11} b_{36}+a_{2} b_{46} b_{71}+a_{3} b_{46} b_{81}+a_{2} b_{51} b_{66}-a_{2} b_{56} b_{61}+a_{5} b_{56} b_{71} \\
& +a_{6} b_{56} b_{81}+a_{7} b_{66} b_{71}+a_{8} b_{66} b_{81}=0 \\
& b_{47}-b_{11} b_{58}-a_{2} b_{51} b_{88}+a_{2} b_{58} b_{81}-\left(a_{3}+2 a_{5}\right) b_{61} b_{88}+\left(a_{3}+2 a_{5}\right) b_{68} b_{81} \\
& -\left(a_{6}+a_{7}\right) b_{71} b_{88}+\left(a_{6}+a_{7}\right) b_{78} b_{81}=0 \\
& a_{8} b_{33}+\left(a_{6}+a_{7}\right) b_{34}+\left(a_{3}+2 a_{5}\right) b_{35}+a_{2} b_{36}-a_{2} b_{47} b_{88}-\left(a_{3}+a_{5}\right) b_{57} b_{88} \\
& -a_{5} b_{67} b_{78}-\left(a_{6}+a_{7}\right) b_{67} b_{88}+a_{5} b_{68} b_{77}-a_{8} b_{77} b_{88}=0 \\
& a_{8} b_{22}+\left(a_{6}+a_{7}\right) b_{23}+\left(a_{3}+2 a_{5}\right) b_{24}+a_{2} b_{25}-a_{2} b_{46} b_{78}-a_{3} b_{46} b_{88} \\
& +a_{2} b_{56} b_{68}-a_{5} b_{56} b_{78}-a_{6} b_{56} b_{88}-a_{2} b_{58} b_{66} \\
& -a_{7} b_{66} b_{78}-a_{8} b_{66} b_{88}=0 \\
& b_{26}-b_{11} b_{37}-a_{2} b_{41} b_{77}+a_{2} b_{47} b_{71}+a_{3} b_{47} b_{81}+a_{2} b_{51} b_{67}-a_{5} b_{51} b_{77} \\
& -a_{2} b_{57} b_{61}+a_{5} b_{57} b_{71}+a_{6} b_{57} b_{81}-a_{7} b_{61} b_{77}+a_{7} b_{67} b_{71} \\
& +a_{8} b_{67} b_{81}+a_{9} b_{77} b_{81}=0 \\
& b_{37}-b_{11} b_{48}-a_{2} b_{41} b_{88}+a_{2} b_{48} b_{81}-\left(a_{3}+a_{5}\right) b_{51} b_{88}+\left(a_{3}+a_{5}\right) b_{58} b_{81} \\
& -a_{5} b_{61} b_{78}-\left(a_{6}+a_{7}\right) b_{61} b_{88}+a_{5} b_{68} b_{71}+\left(a_{6}+a_{7}\right) b_{68} b_{81} \\
& -a_{8} b_{71} b_{88}+a_{8} b_{78} b_{81}=0 \\
& a_{9} b_{22}+a_{8} b_{23}+\left(a_{6}+a_{7}\right) b_{24}+\left(a_{3}+2 a_{5}\right) b_{25}+a_{2} b_{26}-a_{2} b_{47} b_{78}-a_{3} b_{47} b_{88} \\
& +a_{2} b_{48} b_{77}+a_{2} b_{57} b_{68}-a_{5} b_{57} b_{78}-a_{6} b_{57} b_{88}-a_{2} b_{58} b_{67}+a_{5} b_{58} b_{77} \\
& -a_{7} b_{67} b_{78}-a_{8} b_{67} b_{88}+a_{7} b_{68} b_{77}-a_{9} b_{77} b_{88}=0 \\
& b_{27}-b_{11} b_{38}-a_{2} b_{41} b_{78}-a_{3} b_{41} b_{88}+a_{2} b_{48} b_{71}+a_{3} b_{48} b_{81}+a_{2} b_{51} b_{68} \\
& -a_{5} b_{51} b_{78}-a_{6} b_{51} b_{88}-a_{2} b_{58} b_{61}+a_{5} b_{58} b_{71}+a_{6} b_{58} b_{81} \\
& -a_{7} b_{61} b_{78}-a_{8} b_{61} b_{88}+a_{7} b_{68} b_{71}+a_{8} b_{68} b_{81} \\
& -a_{9} b_{71} b_{88}+a_{9} b_{78} b_{81}=0 \text {. }
\end{aligned}
$$


The solutions satisfying $S_{t}^{\prime}(5.6)$ are elements of the set of matrices $B=$ $\left(b_{i j}\right) \in \operatorname{Mat}(8 \times 8, C)$. In case that $B$ are matrices of the form

$$
B=\left(b_{i j}\right) \in \operatorname{Mat}(8 \times 8, C), \quad b_{i j}=0, j<i, b_{i i}=1,
$$

the group of automorphisms $\operatorname{Aut}\left(g_{t}\right)$ of $g_{t}$ consists of unipotent automorphisms.

We will prove that the set of points $t \in V \subset C^{7}$, such that there exists a solution $B=\left(b_{i j}\right) \in \operatorname{Mat}(8 \times 8, C)$ of $S_{t}^{\prime}$ satisfying the conditions (5.7), is a Zariski constructible set and we will express it as a finite union of Zariski locally closed subsets.

To realize the above idea, we study $S_{t}^{\prime}$ in suitable subsets of $V$.

5.2. Main results. We consider two cases: first, $a_{2} \neq 0$ and then, $a_{2}=0$.

5.2.1. $a_{2} \neq 0$. Let the open set $V \cap D\left(a_{2}\right)$. Because of the equation $a_{2}\left(5 a_{5}+\right.$ $\left.2 a_{3}\right)=0$, we can distinguish the following two subcases.

(1) $\left(a_{3} \neq 0\right)$. First, we consider the set $T^{(1)}=V \cap D\left(a_{2} \cdot a_{3}\right)$. From $5 a_{5}+2 a_{3}=$ 0 , we obtain $a_{5}=-(2 / 5) a_{3}$. By doing the necessary calculations in $S_{t}^{\prime}$, we can deduce

$$
b_{11}=b_{88}^{2}, \quad b_{22}=b_{88}^{9}, \quad b_{i i}=b_{88}^{10-i}, \quad i=3, \ldots, 7, \quad Q_{4}\left(b_{88}-1\right)=0
$$

with $Q_{4}=2 a_{3}^{2}-25 a_{2} a_{6}-25 a_{2} a_{7}$.

So, the set of points in $T^{(1)}$ in which the group $\operatorname{Aut}\left(g_{t}\right)$ of the corresponding Lie algebra consists of unipotent automorphisms is $T^{(1)} \cap D\left(Q_{4}\right)$.

(2) $\left(a_{3}=0\right)$. Now, we consider the set $T^{(2)}=V \cap D\left(a_{2}\right) \cap \mathscr{V}\left(a_{3}\right)$. From $5 a_{5}+$ $2 a_{3}=0$, we obtain $a_{5}=0$. By doing some calculations as above, in case that $a_{6}+a_{7} \neq 0$, we deduce

$$
b_{11}=b_{88}^{3}, \quad b_{22}=b_{88}^{11}, \quad b_{i i}=b_{88}^{11-i}, \quad i=3, \ldots, 7, \quad a_{8}\left(b_{88}-1\right)=0 .
$$

So, in the set $T^{(2)} \cap D\left(\left(a_{6}+a_{7}\right) \cdot a_{8}\right)$, the group Aut $\left(g_{t}\right)$ of only one Lie algebra consists of unipotent automorphisms. On the other hand, the group $\operatorname{Aut}\left(g_{t}\right)$ of each of the corresponding Lie algebras in the set $T^{(2)} \cap D\left(a_{6}+a_{7}\right) \cap$ $\mathscr{V}\left(a_{8}\right)$ and $T^{(2)} \cap \mathscr{V}\left(a_{6}+a_{7}\right)$ do not contain unipotent automorphisms.

From the above, we can state the following theorem.

THEOREM 5.1. Consider the set of complex filiform Lie algebras. Consider $C^{8}$ with $\left(a_{2}, a_{3}, \ldots, a_{9}\right)$ as coordinates given by (3.1) and let $V$ be the hypersurface defined in $C^{7}$ by (3.2). In the Zariski open set $V \cap D\left(a_{2}\right)$, the Zariski constructible 
subset of filiform Lie algebras whose group of automorphisms consists of unipotent automorphisms is defined as the union of the following subsets:

$$
\begin{gathered}
D\left(a_{3} \cdot\left(2 a_{3}^{2}-25 a_{2} a_{6}-25 a_{2} a_{7}\right)\right), \\
\mathscr{V}\left(a_{3}\right) \cap D\left(\left(a_{6}+a_{7}\right) \cdot a_{8}\right) .
\end{gathered}
$$

5.2.2. $a_{2}=0$. We consider the set $T^{(3)}=V \cap \mathscr{V}\left(a_{2}\right)$. Because of the equation $a_{2}\left(5 a_{5}+2 a_{3}\right)=0$, we can distinguish the following subcases.

(1) $\left(a_{5} \neq 0\right)$. So, we obtain the set $T^{(3)} \cap D\left(a_{5}\right)$ and we distinguish the following:

(1A) $\left(a_{3}+2 a_{5} \neq 0\right)$. In the subset $T^{(3)} \cap D\left(a_{5} \cdot\left(a_{3}+2 a_{5}\right)\right)$, after the necessary calculations in system (5.6), we deduce

$$
b_{i i}=b_{11}^{10-i}, \quad i=2, \ldots, 8, \quad Q_{5}\left(b_{11}-1\right)=0
$$

with $Q_{5}=2 a_{3}^{2} a_{7}-3 a_{3} a_{5} a_{6}+5 a_{3} a_{5} a_{7}-3 a_{5}^{2} a_{6}+5 a_{5}^{2} a_{7}$.

So, in the set of points $T^{(3)} \cap D\left(a_{5} \cdot\left(a_{3}+2 a_{5}\right) \cdot Q_{5}\right)$, the group $\operatorname{Aut}\left(g_{t}\right)$ of the corresponding Lie algebra consists of unipotent automorphisms, whereas, in the set $T^{(3)} \cap D\left(a_{5} \cdot\left(a_{3}+2 a_{5}\right)\right) \cap \mathscr{V}\left(Q_{5}\right)$, the group Aut $\left(g_{t}\right)$ of the corresponding Lie algebra does not contain unipotent automorphisms.

(1B) $\left(a_{3}+2 a_{5}=0\right)$. We study $S_{t}^{\prime}$ in $T^{(3)} \cap D\left(a_{5}\right) \cap \mathscr{V}\left(a_{3}+2 a_{5}\right)$ and we obtain

$$
b_{i i}=b_{11}^{10-i}, \quad i=2, \ldots, 8, \quad\left(a_{6}+a_{7}\right)\left(b_{11}-1\right)=0 .
$$

So, the group Aut $\left(g_{t}\right)$ of the corresponding Lie algebra in the set of points $T^{(3)} \cap D\left(a_{5} \cdot\left(a_{6}+a_{7}\right)\right) \cap \mathscr{V}\left(a_{3}+2 a_{5}\right)$ consists of unipotent automorphisms, but the group $\operatorname{Aut}\left(g_{t}\right)$ of the Lie algebra corresponding to the set $T^{(3)} \cap D\left(a_{5}\right) \cap$ $\mathscr{V}\left(a_{3}+2 a_{5}, a_{6}+a_{7}\right)$ does not contain unipotent automorphisms.

(2) $\left(a_{5}=0\right)$. First, we distinguish two subcases $a_{3} \neq 0$ and $a_{3}=0$.

(2A) $\left(a_{3} \neq 0\right)$. Then, we consider the set $T^{(3)} \cap \mathscr{V}\left(a_{5}\right) \cap D\left(a_{3}\right)$. By doing some calculations in $S_{t}^{\prime}$, we obtain

$$
b_{i i}=b_{11}^{10-i}, \quad i=2, \ldots, 8, \quad a_{7}\left(b_{11}-1\right)=0 .
$$

In this case, the group $\operatorname{Aut}\left(g_{t}\right)$ of the Lie algebra corresponding to the set of points $T^{(3)} \cap \mathscr{V}\left(a_{5}\right) \cap D\left(a_{3} \cdot a_{7}\right)$ consists of unipotent automorphisms.

(2B) $\left(a_{3}=0\right)$. In $T^{(3)} \cap \mathscr{V}\left(a_{3}, a_{5}\right)$, we distinguish the cases $a_{7} \neq 0$ and $a_{7}=0$.

(i) $\left(a_{7} \neq 0\right)$. In the subset $T^{(3)} \cap \mathscr{V}\left(a_{3}, a_{5}\right) \cap D\left(a_{7}\right)$, after the necessary calculations in system $S_{t}^{\prime}$, we deduce

$$
b_{i i}=b_{11}^{11-i}, \quad i=2, \ldots, 8, \quad a_{8}\left(b_{11}-1\right)=0 .
$$

So, the group Aut $\left(g_{t}\right)$ of the Lie algebra corresponding to the set of points $T^{(3)} \cap \mathscr{V}\left(a_{3}, a_{5}\right) \cap D\left(a_{7} \cdot a_{8}\right)$ consists of unipotent automorphisms. 
(ii) $\left(a_{7}=0\right)$. We now consider the subset $T^{(3)} \cap \mathscr{V}\left(a_{3}, a_{5}, a_{7}\right)$. We distinguish another two subcases $a_{6} \neq 0$ and $a_{6}=0$.

(iiA) $\left(a_{6} \neq 0\right)$. So, we obtain the set $T^{(3)} \cap \mathscr{V}\left(a_{3}, a_{5}, a_{7}\right) \cap D\left(a_{6}\right)$. By doing some calculations in $S_{t}^{\prime}$, we deduce

$$
b_{i i}=b_{11}^{11-i}, \quad i=2, \ldots, 8, \quad a_{8}\left(b_{11}-1\right)=0 .
$$

From the above, we conclude that the group $\operatorname{Aut}\left(g_{t}\right)$ of the Lie algebra corresponding to $T^{(3)} \cap \mathscr{V}\left(a_{3}, a_{5}, a_{7}\right) \cap D\left(a_{6} \cdot a_{8}\right)$ consists of unipotent automorphisms, but the groups Aut $\left(g_{t}\right)$ of those that correspond to $T^{(3)} \cap \mathscr{V}\left(a_{3}, a_{5}, a_{7}\right.$, $\left.a_{8}\right) \cap D\left(a_{6}\right)$ do not contain unipotent automorphisms.

(iiB) $\left(a_{6}=0\right)$. The set of points in which we are acting now is $T^{(3)} \cap \mathscr{V}\left(a_{3}, a_{5}\right.$, $\left.a_{6}, a_{7}\right)$. In case that $a_{8} \neq 0$ and by using similar techniques as we did previously in system $S_{t}^{\prime}$, we deduce

$$
b_{i i}=b_{11}^{12-i}, \quad i=2, \ldots, 8, \quad a_{9}\left(b_{11}-1\right)=0 .
$$

Hence, the group Aut $\left(g_{t}\right)$ of the Lie algebra in the set $T^{(3)} \cap \mathscr{V}\left(a_{3}, a_{5}, a_{6}, a_{7}\right) \cap$ $D\left(a_{8} \cdot a_{9}\right)$ consists of unipotent automorphisms. On the other hand, the groups $\operatorname{Aut}\left(g_{t}\right)$ of each of the algebras in the subsets $T^{(3)} \cap \mathscr{V}\left(a_{3}, a_{5}, a_{6}, a_{7}, a_{9}\right) \cap$ $D\left(a_{8}\right), T^{(3)} \cap \mathscr{V}\left(a_{3}, a_{5}, a_{6}, a_{7}, a_{8}\right) \cap D\left(a_{9}\right)$, and $T^{(3)} \cap \mathscr{V}\left(a_{3}, a_{5}, a_{6}, a_{7}, a_{8}, a_{9}\right)$ do not contain unipotent automorphisms.

So, we have proved the following theorem.

THEOREM 5.2. Consider the set of complex filiform Lie algebras. Consider $C^{8}$ with $\left(a_{2}, a_{3}, \ldots, a_{9}\right)$ as coordinates given by (3.1) and let $V$ be the hypersurface defined in $C^{7}$ by (3.2). The Zariski constructible subset of filiform Lie algebras whose group of automorphisms consists of unipotent automorphisms in the Zariski closed set $V \cap \mathscr{V}\left(a_{2}\right)$ is defined as the union of the following subsets:

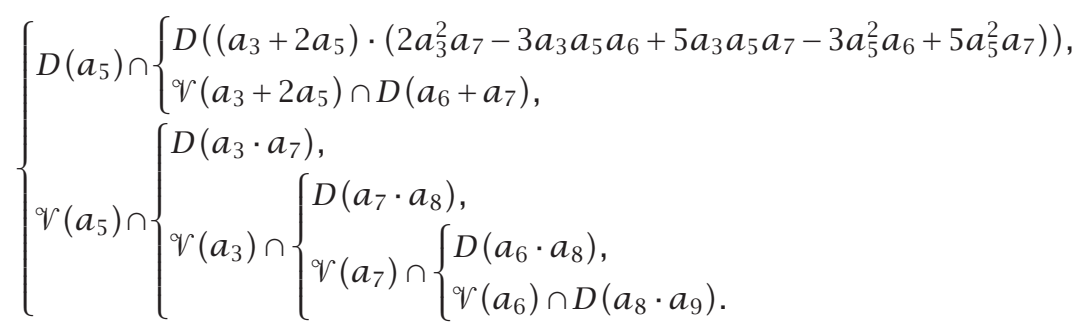

$B y(\{)$, we mean the union of the corresponding sets. 
Let $g_{1}=\mu_{8}^{10, a}$ and $g_{2}=\mu_{8}^{11}$ be the following Lie algebras belonging to the family (3.1) as they were defined in [1]:

$$
\begin{array}{rlrl}
{\left[e_{1}, e_{i}\right]} & =e_{i-1}, \quad i \geq 3, & & {\left[e_{4}, e_{8}\right]=e_{2},} \\
{\left[e_{5}, e_{8}\right]} & =e_{3}, & & {\left[e_{6}, e_{8}\right]=e_{2}+e_{4},} \\
{\left[e_{7}, e_{8}\right]} & =a e_{2}+e_{3}+e_{5}, \quad a \in C, & \\
{\left[e_{1}, e_{i}\right]} & =e_{i-1}, \quad i \geq 3, & \\
{\left[e_{i}, e_{8}\right]} & =e_{i-2}, \quad 4 \leq i \leq 6, & {\left[e_{7}, e_{8}\right]=e_{2}+e_{5} .}
\end{array}
$$

Now, we can state the following theorems.

THEOREM 5.3. Consider the set of complex filiform Lie algebras. If $C^{8}=$ $\left\{\left(a_{2}, \ldots, a_{9}\right) / a_{i} \in C\right.$ and $a_{i}$ satisfy (3.1)\}, we define the hypersurface $V$ in $C^{7}$ by (3.2). The group $\operatorname{Aut}\left(g_{1}\right)$ of $g_{1}$ corresponding to the set $V \cap \mathscr{V}\left(a_{2}, a_{5}, a_{7}\right) \cap$ $D\left(a_{3} \cdot\left(4 a_{3} a_{8}-5 a_{6}^{2}\right)\right)$ consists of automorphisms of the type

$$
\begin{aligned}
& L=\left(l_{i j}\right), \quad l_{i j}=0, j<i, l_{i i}=1,1 \leq i, j \leq 8 . \\
& L=\left(l_{i j}\right), \quad l_{i j}=0, j<i, l_{i i}= \begin{cases}1, & i \text { is even, } \\
-1, & i \text { is odd },\end{cases}
\end{aligned}
$$

where $1 \leq i, j \leq 8$.

So, the set of unipotent automorphisms form a proper subgroup of the group $\operatorname{Aut}\left(g_{1}\right)$.

Proof. If we consider the set of points $V \cap \mathscr{V}\left(a_{2}, a_{5}, a_{7}\right) \cap D\left(a_{3}\right)$ after the necessary calculations in system $S_{t}^{\prime}$, we deduce

$$
b_{i i}=b_{11}^{10-i}, \quad i=2, \ldots, 8, \quad\left(4 a_{3} a_{8}-5 a_{6}^{2}\right)\left(b_{11}^{2}-1\right)=0 .
$$

Obviously, the group $\operatorname{Aut}\left(g_{1}\right)$ of the Lie algebras corresponding to the set of points $V \cap \mathscr{V}\left(a_{2}, a_{5}, a_{7}\right) \cap D\left(a_{3} \cdot\left(4 a_{3} a_{8}-5 a_{6}^{2}\right)\right)$ does not contain only unipotent automorphisms. So, the group $\operatorname{Aut}\left(g_{1}\right)$, except the unipotent, contains automorphisms of the following type:

$$
L=\left(l_{i j}\right), \quad l_{i j}=0, j<i, l_{i i}= \begin{cases}1, & i \text { is even } \\ -1, & i \text { is odd }\end{cases}
$$

where $1 \leq i, j \leq 8$. 
In case that $4 a_{3} a_{8}-5 a_{6}^{2}=0$, by acting as we previously did in system $S_{t}^{\prime}$, we obtain

$$
b_{i i}=b_{11}^{10-i}, \quad i=2, \ldots, 8, \quad\left(4 a_{3}^{2} a_{9}-7 a_{6}^{3}\right)\left(b_{11}^{3}-1\right)=0 .
$$

Thus, the group Aut $\left(g_{2}\right)$ of the Lie algebra corresponding to the set of points $V \cap \mathscr{V}\left(a_{2}, a_{5}, a_{7}, 4 a_{3} a_{8}-5 a_{6}^{2}\right) \cap D\left(a_{3} \cdot\left(4 a_{3}^{2} a_{9}-7 a_{6}^{3}\right)\right)$ does not contain only unipotent automorphisms but also automorphisms of the type

$$
K=\left(k_{i j}\right), \quad k_{i j}=0, j<i, k_{i i}= \begin{cases}z, & i=1,3,6, \\ \bar{z}, & i=2,5,8, \\ 1, & i=4,7,\end{cases}
$$

where $j=1, \ldots, 8$ and $z$ is a cubic root of 1 .

So, we have proved the following theorem.

THEOREM 5.4. Consider the set of complex filiform Lie algebras. If $C^{8}=$ $\left\{\left(a_{2}, \ldots, a_{9}\right) / a_{i} \in C\right.$ and $a_{i}$ satisfy (3.1)\}, we define the hypersurface $V$ in $C^{7}$ by (3.2). The group Aut $\left(g_{2}\right)$ of the filiform Lie algebra $g_{2}$ corresponding to the set $V \cap \mathscr{V}\left(a_{2}, a_{5}, a_{7}, 4 a_{3} a_{8}-5 a_{6}^{2}\right) \cap D\left(a_{3} \cdot\left(4 a_{3}^{2} a_{9}-7 a_{6}^{3}\right)\right)$ consists of automorphisms of the type

$$
K=\left(k_{i j}\right), \quad k_{i j}=0, j<i, k_{i i}= \begin{cases}z, & i=1,3,6 \\ \bar{z}, & i=2,5,8 \\ 1, & i=4,7\end{cases}
$$

where $j=1, \ldots, 8$ and $z$ is a cubic root of 1 .

REMARK 5.5. The group Aut $(g)$ of the Lie algebra corresponding to the set of points $V \cap \mathscr{V}\left(a_{2}, a_{5}, a_{7}, 4 a_{3} a_{8}-5 a_{6}^{2}, 4 a_{3}^{2} a_{9}-7 a_{6}^{3}\right) \cap D\left(a_{3}\right)$ does not contain unipotent automorphisms.

6. General conclusions. From Theorems 4.1, 4.2, 5.1, 5.2, 5.3, and 5.4, we conclude.

THEOREM 6.1. The group of automorphisms Aut $(g)$ of each one of the characteristically nilpotent filiform Lie algebras of dimension 8 over $C$ given by (4.6) and (4.7) consists of unipotent automorphisms, except that of the Lie algebras $g_{1}=\mu_{8}^{10, a}, a \in C$, and $g_{2}=\mu_{8}^{11}$ given by (5.18), and (5.19).

THEOREM 6.2. The unipotent automorphisms of each one of the characteristically nilpotent filiform Lie algebras of dimension 8 over $C, g_{1}=\mu_{8}^{10, a}, a \in C$, 
and $g_{2}=\mu_{8}^{11}$, given by (5.18) and (5.19), form a proper subgroup of the group $\operatorname{Aut}\left(g_{1}\right)$ and $\operatorname{Aut}\left(g_{2}\right)$, respectively.

THEOREM 6.3. The group of automorphisms Aut $\left(g_{1}\right)$ of the characteristically nilpotent filiform Lie algebras of dimension 8 over $C, g_{1}=\mu_{8}^{10, a}, a \in C$, given by (5.18), consists of automorphisms of the type

$$
\begin{aligned}
& L=\left(l_{i j}\right), \quad l_{i j}=0, j<i, l_{i i}=1,1 \leq i, j \leq 8, \\
& L=\left(l_{i j}\right), \quad l_{i j}=0, j<i, l_{i i}= \begin{cases}1, & i \text { is even, } \\
-1, & i \text { is odd },\end{cases}
\end{aligned}
$$

where $1 \leq i, j \leq 8$.

THEOREM 6.4. The group of automorphisms Aut $\left(g_{2}\right)$ of the characteristically nilpotent filiform Lie algebra of dimension 8 over $C, g_{2}=\mu_{8}^{11}$, given by (5.19), consist of automorphisms of the type

$$
K=\left(k_{i j}\right), \quad k_{i j}=0, j<i, k_{i i}= \begin{cases}z, & i=1,3,6, \\ \bar{z}, & i=2,5,8, \\ 1, & i=4,7,\end{cases}
$$

where $j=1, \ldots, 8$ and $z$ is a cubic root of 1 .

Acknowledgment. We wish to express our deep thanks to Professors G. Thorbergsson and F. Xenos for their very useful advice and help.

\section{REFERENCES}

[1] J. M. Ancochéa-Bermúdez and M. Goze, Classification des algèbres de Lie filiformes de dimension 8 [Classification of filiform Lie algebras of dimension 8], Arch. Math. (Basel) 50 (1988), no. 6, 511-525 (French).

[2] F. J. Castro-Jiménez and J. Núñez-Valdés, On characteristically nilpotent filiform Lie algebras of dimension 9, Comm. Algebra 23 (1995), no. 8, 3059-3071.

[3] _ Gröbner basis in the classification of characteristically nilpotent filiform Lie algebras of dimension 10, Algorithms in Algebraic Geometry and Applications (Santander, 1994), Progr. Math., vol. 143, Birkhäuser, Basel, 1996, pp. 115-133.

[4] J. Dixmier and W. G. Lister, Derivations of nilpotent Lie algebras, Proc. Amer. Math. Soc. 8 (1957), 155-158.

[5] J. L. Dyer, A nilpotent Lie algebra with nilpotent automorphism group, Bull. Amer. Math. Soc. 76 (1970), 52-56.

[6] F. J. Echarte-Reula, J. R. Gómez-Martin, and J. Núñez-Valdés, On complex filiform Lie algebras derived or not derived from other Lie algebras, Geometry and Topology, Proceedings of the 15th Portuguese-Spanish Conference on Mathematics, Vol. III (Portuguese) (Évora, 1990), Univ. Évora, Évora, 1991, pp. 101-106.

[7] G. Favre, Une algèbre de Lie caractéristiquement nilpotente de dimension 7, C. R. Acad. Sci. Paris Sér. A-B 274 (1972), A1338-A1339 (French). 
[8] M. Vergne, Sur la variété des algèbres de Lie nilpotentes, Thèse de 3ème cycle, Paris, 1966.

P. Barbari: Department of Mathematics and Physics, School of Technology, Aristotle University of Thessaloniki, Thessaloniki 540 06, Greece

E-mail address: pbar@vergina.eng.auth.gr

A. Kobotis: Department of Business Administration, University of Macedonia, 156 Egnatia Street, Thessaloniki 540 06, Greece

E-mail address: kompotis@macedonia.uom.gr 


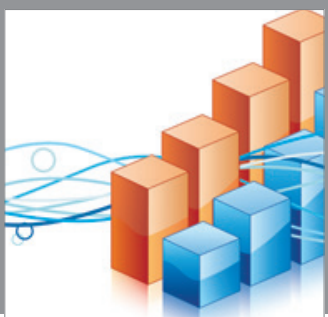

Advances in

Operations Research

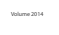

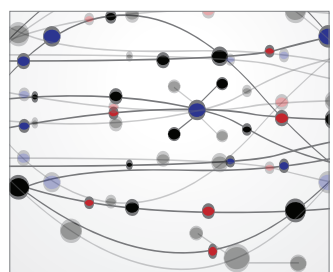

\section{The Scientific} World Journal
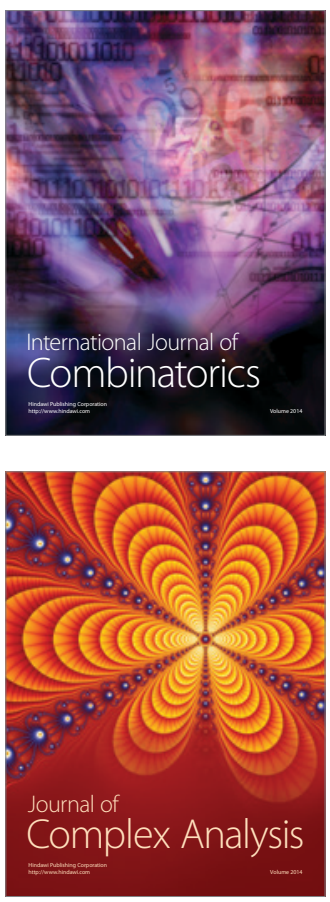

International Journal of

Mathematics and

Mathematical

Sciences
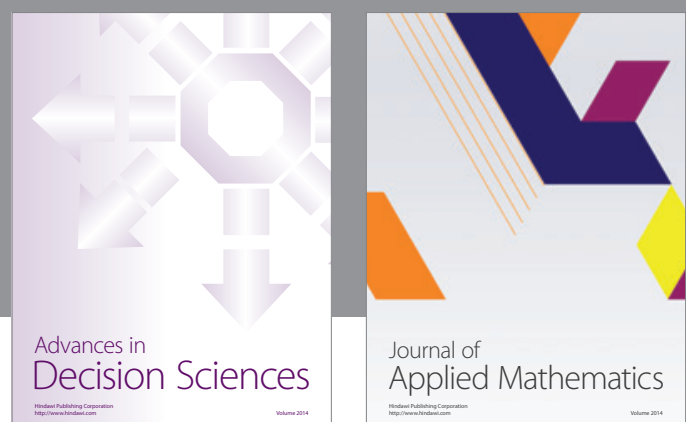

Journal of

Applied Mathematics
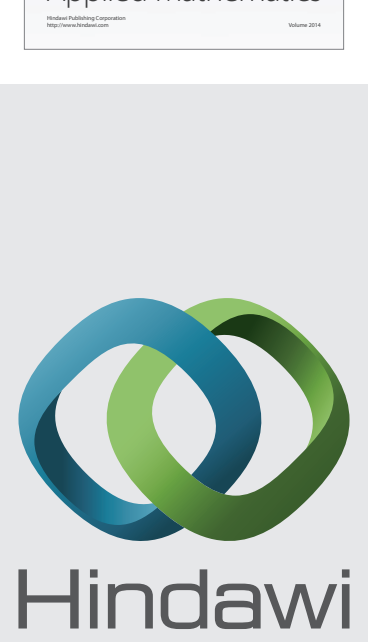

Submit your manuscripts at http://www.hindawi.com
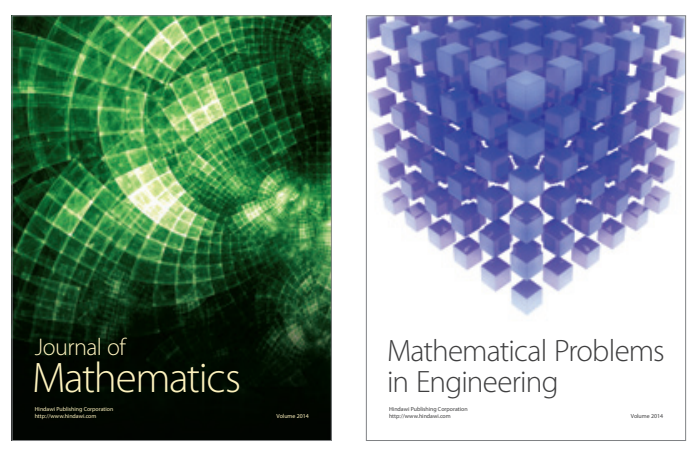

Mathematical Problems in Engineering
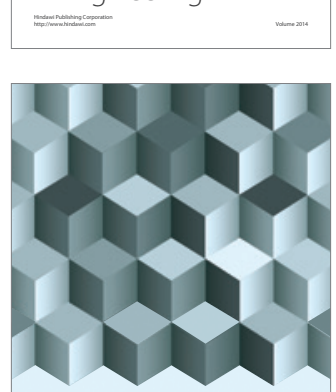

Journal of

Function Spaces


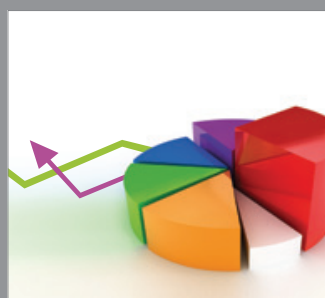

ournal of

Probability and Statistics

Promensencen
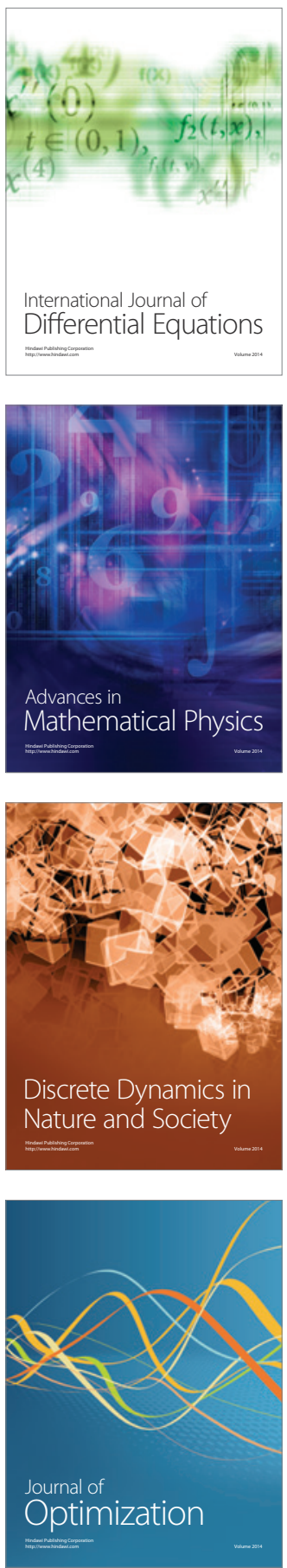